\title{
Effet de l'addition d'un conservateur biologique sur la qualité de l'ensilage en balles rondes enrubannées
}

\author{
E Viviani Rossi 1, LM Gutierrez 1, E Delpech 3, L Matinata 1, E Moreno 2 \\ 1Facultad de Ciencias Agrarias, Universidad Nacional de Mar del Plata ; ${ }^{2}$ Agente de proyecto, \\ Instituto Nacional de Tecnología Agropecuaria; ; Unidad Integrada (FCA, UNMDP - EEA Balcarce, \\ INTA), Area de Producción Animal, CC 276, 7620 Balcarce, Argentina
}

L'enrubannage est une technique de conservation de fourrages humides adoptée récemment par les systèmes de production intensive de lait et de viande dans la province de Buenos Aires en Argentine. Dans ces systèmes, la qualité du fourrage conservé est primordiale pour l'alimentation du troupeau durant toute l'année. Cette technique permet une récolte des fourrages plus précoce que le foin.

L'effet de l'addition d'un conservateur biologique à l'ensilage en balles rondes enrubannées reste à étudier.

L'essai a été réalisé avec un fourrage de pairie temporaire humide [Phalaris aquatica, $75 \%$; Festuca arundinacea, $16 \%$; Lolium perenne, $5 \%$; Medicago sativa, $4 \%$ de la matière sèche (MS)]. Les caractéristiques du fourrage lors de l'enrubannage sont : \% MS, $37 \%$, digestibilité in vitro de la MS (DMS) selon Tilley et Terry $60,4 \%$; matières azotées (MAT) $12 \%$ et NDF de Van Soest $60 \%$ de la MS. Un conservateur biologique à base de ferments lactiques ( $L$. plantarum, $S$. faecium et $P$. acidilactici), 118 billions CFU/lb et d'enzymes (cellulase, hémicellulases et amylase) a été utilisé à la dose de $5 \mathrm{~g} / \mathrm{t}$ de fourrage vert. La pression de travail de la presse à balles rondes a été de $80 \mathrm{~kg} / \mathrm{cm}^{2}$ et on a pris soin de ne pas contaminer le fourrage avec de la terre. Les balles ont été enrubannées avec 4 couches d'un fitm blanc de 25 microns d'épaisseur, étiré de $50 \%$. Dix balles ont été échantillonnées au moment de la distribution aux animaux (80 jours après l'enrubannage).

Les résultats (cf tableau) montrent qu'à la dose commercialement recommandée le conservateur biologique n'a pas amélioré la qualité de conservation qui peut être considérée comme satisfaisante selon Dulphy et Demarquilly (1978, INRA Theix).

\begin{tabular}{|c|c|c|c|c|c|c|c|}
\hline Balles rondes ensilées & $\begin{array}{c}\text { DMS } \\
(\% \mathrm{MS})\end{array}$ & $\begin{array}{c}\text { MAT } \\
(\% \mathrm{MS})\end{array}$ & $\begin{array}{c}\text { NDF } \\
(\% \mathrm{MS})\end{array}$ & $\mathrm{pH}$ & $\begin{array}{l}\text { MS } \\
(\%)\end{array}$ & $\begin{array}{c}\mathrm{N}-\mathrm{sol} \% \\
\mathrm{~N} \text { total }\end{array}$ & $\begin{array}{c}\mathrm{N}-\mathrm{NH}_{3} \% \\
\mathrm{~N} \text { total }\end{array}$ \\
\hline avec conservateur & 61,5 & 11,95 & 60,3 & 4,35 & 36,5 & 73,3 & 11,5 \\
\hline sans conservateur & 61,5 & 11,9 & 60,7 & 5,0 & 32,0 & 73,3 & 12,0 \\
\hline
\end{tabular}

Ce travail a été financé par l'UNMDP et l'INTA. 